\title{
MICHEL FOUCAULT, L'HERMENEUTIQUE, ET LA CRITIQUE DES LUMIERES
}

La désintégration de la modernité se trouve confirmée d'une façon révélatrice par le discrédit qui invalide aujourd'hui la fameuse recommandation de Marx aux philosophes. Les philosophes, selon Marx, n'avaient fait qu'interpréter le monde alors qu'il importait surtout de le changer. La célèbre formule se trouve invertie aujourd'hui. Les transformations que le monde subit se sont avérées si profondes et si imprévisibles que toute suggestion proposant la possibilité de contrôler le réel ne peut qu'apparaître naïve à présent. Dans un sens plus large, c'est tout le projet des Lumières---ses promesses de progrès et d'emancipation qui est mis en cause et loin de vouloir prétendre changer le monde, les philosophes ont plus que jamais des raisons qui les maintiennent dans les limites beaucoup plus modestes de l'interprétation.

En même temps, l'interprétation acquiert une importance nouvelle, due en grande partie à son rôle politique. Plus précisément, l'interprétation a servi à démystifier la question du politique, ainsi que l'observe Paul Ricoeur: "Le service du philosophe dans cette question, c'est de montrer d'une part que la politique, comme brigue du pouvoir, n'épuise pas le politique comme structure de la réalité humaine; et d'autre part que le politique n'épuise pas tout le champ anthropologique" (VI, 156). Depuis que les supports ontologiques du politique ont été abolis, le politique se trouve relégué à une position subalterne, inféodé à la stratégie herméneutique qui se donne pour but de clarifier les contextes de l'interprétation. Comme le montre Barry Allen dans son étude Truth in Philosophy.

\footnotetext{
With being, presence, and self-identical unity deferred ad infinitum, the value of truth can and must be "reinscribed" where it belongs: "in more powerful, larger, more stratified...interpretative contexts"----in other words, in the institutional-social practice where truth always was anyway, even when philosophers preferred to avert their eyes from time, history, and contingency and dream of True Being (I, 111).
}

Ces "projets de réinscription dans les contextes interprétatifs" nous fournissent une bonne définition de ce qui constitue le travail de bon 
nombre de penseurs aujourd'hui. C'est ainsi que la société a remplacé la nature comme cadre de référence. La vérité du sujet ne se rapportant plus à la vérité unifiante de la nature, elle se trouve fragmentée à la mesure des processus d'intégration sociale et d'adaptation culturelle. Le sujet se trouve ainsi soumis à des effets et des forces qui le structurent selon des logiques hétérogènes et autonomes. Il ne s'agit plus, par conséquent, de comprendre les actions du sujet à partir d'une vérité intérieure, d'une auto-représentation ou d'une auto-reconstitution, mais d'analyser la constitution du sujet du point de vue de son expérience. Ce point de vue permet d'apprécier le rapport du sujet au système qui l'intègre aussi bien que de voir la distance qui les sépare l'un de l'autre. Et c'est en mesurant cette distance qu'il devient possible de déceler les subterfuges soutenant les stratégies de domination. Ainsi l'idée du sujet maître de lui et de la nature a joué un rôle stratégique d'assujettissement puisque, comme nous le dit le sociologue François Dubet, "l'autonomie de l'acteur est une illusion, elle est la figure même du pouvoir et de la domination" (II, 14). C'est ce que démontre la critique postnietzschéenne de la modernité, selon Dubet: le règne de la Raison s'y trouve associé "à la formation de nouveaux dispositifs de pouvoirs. Le sujet libre de la raison ne devient lui-même que la représentation du pouvoir intériorisée dans l'idée du sujet autonome, dans la croyance en la réalité d'un individu maître de son Moi. Le grand récit de la modernité et de l'évolution n'est plus celui de la libération et de la connaissance, mais c'est l'histoire de l'assujettissement" (II, 55). Cette histoire peut se comprendre de diverses façons. Ainsi, pour une certaine approche herméneutique remontantà Heidegger, la situation dépendante du sujet se laisse approcher par le biais d'une notion de la compréhension. Le sujet est tout d'abord un sujet de compréhension-ou, plus précisément, il est sujet à une situation de compréhension qui est antérieure à tout effort de compréhension: "Selon Heidegger, il n'y a pas d'abord des choses objectives dans un monde exterieur, là-dehors, qui recevraient ensuite de notre compréhension prévoyante une coloration subjective ou pragmatiste. Non, ce qui est d'abord là, c'est notre rapport au monde sur le mode de projets anticipants de compréhension" (IV, 136). Le statut de l'interprétation change, inévitablement, puisque l'interprétation "consistera seulement à élaborer ou élucider, après coup, la situation de compréhension" (IV, 137). Pour Gadamer, qui poussera plus loin dans la voie indiquée par Heidegger, cette situation est marquée par son historicité puisque ce n'est pas l'histoire qui nous appartient mais nous, nous appartenons bien à l'histoire. C'est pourquoi, selon Gadamer, "Les préjugés de l'individu, bien plus que ses jugements, constituent la 
réalité historique de son être" (IV, 174). Même la raison que nous prétendons utiliser pour dissoudre nos préjugés est secondaire aux processus qui l'ont formée. Par conséquent, selon Gadamer, la tâche essentielle du philosophe est herméneutique et consiste à établir, non pas ce que nous faisons ou ce que nous devrions faire, mais ce qui nous arrive au delà de notre vouloir et de notre faire. C'est ici que la position de Gadamer marque le plus clairement son affinité à une certaine pensée post-structuraliste, ainsi que l'ont déjà fait remarquer plusieurs critiques.

Selon Susan Hekman, par exemple, "It is Gadamer's tendency to decenter 'man' and his rejection of the epistemological dichotomies of Enlightenment thought that form the bridge between Gadamer and poststructuralist thinkers." La pensée de Michel Foucault semble être remarquablement proche de certains aspects de l'herméneutique gadamérienne; Hekman souligne en particulier "The explicit anti-foundationalism" qui constitue, selon elle, le lien le plus significatif entre les deux philosophes (V. 171 and 181). En même temps, Hekman considère la méthode de Gadamer supérieure à celle du philosophe français parce que le soi-disant nihilisme Nietzschéen de Foucault empêche celui-ci de développer une position morale et, par conséquent, d'offrir une base positive à l'action politique ( $\mathrm{V}, 185)$. Remarquons, entre parenthèses, que ce dernier reproche est parmi les plus communs qu'on ait faits à Foucault. Selon Hekman, donc, le défaut principal de la méthode foucaldienne est d'avoir éliminé la catégorie du sujet. Là aussi, il faut le dire, nous avons un reproche qui revient assez fréquemment sous la plume des critiques de Foucault. C'est un reproche, d'autre part, qui pourrait bien surprendre, puisqu'il est évident que la notion de sujet est centrale dans l'oeuvre de Foucault. Essayons donc d'y voir plus clair.

Il est évident que Foucault rejette le sujet, ou, plus précisément, qu'il désire se passer de la perspective à laquelle nous oblige le thème d'un sujet fondateur. Pour Foucault, il s'agit d'un choix qui s'impose dans le contexte de son approche généalogique: il faut choisir entre deux points de vue inconciliables---celui du sujet ou du discours. Il précise les raisons derrière ce choix dans un essai examinant la fameuse question de Kant au sujet des Lumières---Was ist Auflärung?"

si la question kantienne était de savoir quelles limites la connaissance doit renoncer à franchir, il me semble que la question critique aujourd'hui doit être retournée en question positive: dans ce qui nous est donné comme universel, nécessaire, obligatoire, quelle est la part de ce qui est singulier, contingent et da à des contraintes arbitraires (IIlc, 562-578). 
Or, c'est justement le sujet qui nous empêche d'apprécier les évènements discursifs dans la singularité et la contingence de leur mode d'être. Selon Foucault, il faut "traiter les discours qui articulent ce que nous pensons, disons et faisons, comme autant d'évènements historiques" (IIIc, 562-578). Ceci permet d'individualiser les discours--ainsi que d'eviter le theme imaginaire de l'individualisation des sujets. La question du sujet se retourne, en même temps vers la position du sujet; dans cette inversion généalogique, le sujet qui prétendait être à l'origine du discours en devient l'objet. Foucault propose donc que nous procédions à ce qu'il appelle une ontologie historique de nous-mêmes, une ontologie qui "doit se détourner de tous ces projets qui prétendent être globaux et radicaux" (IIIc, 575). Ces projets sont à éviter parce qu'ils se fondent sur des valeurs ayant l'apparence de l'universel, du nécessaire, de ce qui est obligatoire. Ces qualifications, à leur tour dérivent de certaines "conceptions de l'homme qui sont empruntées à la religion, à la science, à la politique" (IIIc, 573), c'est-à-dire à certaines pratiques discursives.

Le phénomène du changement se présente ainsi d'une manière entièrement inédite---non plus en termes de causes imaginaires attribuées aux agissements de sujets pleinement conscients et volontaires mais du point de vue du contenu du changement. C'est pourquoi, précise Foucault dans un article bien connu intitulé "Réponse à une question," il est important de "définir avec le plus grand soin les transformations qui ont, je ne dis pas: provoqué, mais constitué le changement" (IIIb, 673-95). Il s'agit de souligner ce qu'il appelle "le fait sauvage du changement" afin de "libérer le champ discursif de la structure historicotranscendantaleque la philosophie du XIXe siecle lui a imposée." L'approche de Foucault, nous le voyons, consiste à aborder le discours en termes d'une dimension qui échappe à une prise de conscience volontaire; le discours est surtout ce qui se dit à l'insu du sujet conscient, "il est constitué, nous dit Foucault, par la différence entre ce qu'on pourrait dire correctement à une époque (selon les règles de la grammaire et celles de la logique) et ce qui est dit effectivement. Le champ discursif, c'est à un moment déterminé, la loi de cette différence" (IIIb, 685). Puisque ce n'est plus l'identité du sujet qui se révèle dans un discours, la tâche de l'historien est de "raconter l'histoire des idées comme l'ensemble des formes spécifiées et descriptives de la non-identité" (IIIb, 685). Tâche paradoxale---et difficile, cela va sans dire, car cette façon de concevoir le discours s'oppose à un parti-pris profond de notre modernité.

Cette invitation de penser qu'"en chaque phrase règne la loi sans nom, la blanche indifférence: 'Qu'importe qui parle; quelqu'un a dit: qu'importe qui parle'" (IIIb, 695) peut en effet sembler déroutante, 
inquiétante, même. Elle comporte des avantages certains, pourtant. Ce qui se révèle en l'absence d'un sujet qui garantisse le sens du discours c'est la dimension herméneutique de toute signification, le fait que "le signe est déjà une interprétation qui ne se donne pas pour telle," que "tout est déjà interprétation, chaque signe est en lui-même non pas la chose qui s'offre à l'interprétation, mais l'interprétation d'autres signes" (IIIa,571-72). L'élimination du sujet sert à révéler que la formation et la trans-formation des "choses dites" suivent leurs propres règles et possèdent une sytématicité qui leur est propre.

Deuxiemement, cette approche permet de faire "une histoire des pratiques discursives dans les rapports spécifiques qui les articulent sur les autres pratiques" (IIIb, 687). Le concept de cette articulation est central à la critique des Lumières élaborée par Foucault. "On sait que la grande promesse ou le grand espoir du $18 \mathrm{e}$ siècle, ou d'une partie du 18e sieccle, était dans la croissance simultanée et proportionnelle de la capacité technique à agir sur les choses et de la liberté des individus les uns par rapport aux autres." Or, il s'est avéré par la suite que "les relations entre croissance des capacités et croissance de l'autonomie ne sont pas aussi simples que le 18e siècle pouvait croire" (IIIc, 575-76). Ce que le siècle n'a pas su prévoir c'est l'opposition qui allait se produire entre la capacité technique et la liberté des individus. C'est pourquoi, selon Foucault, le rôle d'une stratégie critique progressiste est de mettre en question "toutes les métaphores dynamiques, biologiques, évolutionnistes par lesquelles on masque le probleme difficile du changement historique" (IIIb, 687) car l'enjeu, justement, c'est de savoir "comment déconnecter la croissance des capacités et l'intensification des relations de pouvoir" (IIIc, 576). C'est aussi dans ce sens qu'une stratégie politique trouvera sa définition: elle consistera à tranformer "les conditions d'existence et les systèmes de fonctionnement du discours" (IIIb, 691). Nous reconnaissons là le travail de l'intellectuel spécifique, tel qu'il a été défini par Foucault, travail qui s'élabore depuis un certain nombre d'années déjà. Je préfere, nous dit Foucault:

les transformations très precises qui ont pu avoir lieu depuis vingt ans dans un certain nombre de domaines qui concernent des modes d'Etre et de penser, les relations d'autorité, les rapports de sexes, la façon dont nous percevons la folie ou la maladie, je prefere ces transformations même partielles qui ont été faites dans la corrélation de l'analyse historique et de l'attitude pratique, aux promesses de l'homme nouveau que les pires systèmes politiques ont répétées au long du XXe siècle (IIIc, 575) 
L'approche foucaldienne est, en fin de compte, une éthique, ainsi que l'ont dejà noté plusieurs critiques. Foucault parle en effet de "l' ethos philosophique propre à l'ontologie critique de nous mêmes," en le définissant "comme une épreuve historico-pratique des limites que nous pouvons franchir, et donc comme travail de nous-mêmes sur nous-mêmes en tant qu'êtres libres" (IIIc, 575).

C'est dans le but de dévoiler et de dévaluer les processus d'assujettissement que le travail de nous-mêmes sur nous-mêmes devient une hermeneutique, une analyse des formations de sens qui nous somment de devenir sujets selon les trois axes definis par Foucault, à savoir "comment nous sommes-nous constitués comme sujets de notre savoir; comment nous sommes-nous constitués comme sujets qui exercent ou subissent des relations de pouvoir; comment nous sommesnous constitués comme sujets moraux de nos actions" (IIIc, 576). Cette approche est, en même temps, généalogique car "elle ne déduira pas de la forme de ce que nous sommes ce qu'il nous est impossible de faire ou de connaître; mais elle dégagera de la contingence qui nous a fait être ce que nous sommes, la possibilité de ne plus être, faire ou penser ce que nous sommes, faisons ou pensons" (IIIc, 574).

Travail critique qui est toujours à recommencer, puisqu'il nous est impossible de comprendre ou de contrôler notre situation de compréhension: "L'expérience théorique et pratique que nous faisons de nos limites et de leur franchissement possible est toujours elle-même limitee, déterminée et donc à recommencer" (IIIc, 575). La raison qui nous sert à dévoiler nos limites est aussi celle qui les impose: cette ambiguité propre au travail de la raison est longtemps restee occultee par une certaine représentation de la pensée des Lumières. Refoulée au nom de divers positivismes, elle émerge de nouveau aujourd'hui, puisque c'est sous le signe de cette ambiguité que s'opère le retour des Lumières, ainsi que Foucault l'observe dans le tout dernier essai qu'il eut l'occasion de réviser avant sa mort prématurée:

Deux siècles après son apparition, l'Aufklärung fait retour. A la fois comme une manière pour l'Occident de prendre conscience de ses possibilités actuelles et des libertés auxquelles il peut avoir accès, mais aussi comme une manière de s'interroger sur ses limites et sur les pouvoirs dont il a usé. La raison à la fois comme despotisme et comme lumière. ${ }^{1}$

${ }^{1}$ Michel Foucault, "La vie: l'expérience et la science," Dits et Écrits, vol. IV, p. 768. La version première de ce passage publié en 1978, était la suivante: "Deux siècles après, l'Aufklärung fait retour: non point comme une maniere pour l'Occident de prendre conscience de ses possibilités actuelles et des libertés auxquelles il peut avoir accès, mais 


\section{BIBLIOGRAPHIE}

I. Allen, Barry. Truth in Philosophy. Cambridge, Mass.: Harvard University Press, 1993.

II. Dubet, François. Sociologie de l'expérience. Paris: Seuil, 1994.

IIIa. Foucault, Michel . Dits et écrits. Vol. I. "Nietzsche, Freud, Marx." Paris: Gailimard, 1994.

IIIb. Vol. I. "Réponse à une question."

IIIc. Vol. IV. "Qu'est-ce que les

IV. Grondin, Jean. L'universalité de l'herméneutique. Paris: PUF, 1993.

V. Hekman, Susan J. Hermeneutics and the Sociology of Knowledge. Notre Dame, Ind.: University of Notre Dame Press, 1986.

VI. Ricoeur, Paul. La critique et la conviction: Entretiens avec François Azouvi et Marc de Launay. Paris: Calmann-Lévy, 1995. 